\title{
Accuracy of the Diagnosis of GORD by Questionnaire, Physicians and a Trial of Proton Pump Inhibitor Treatment: The Diamond Study
} (Gut 2010;59:714-721)

Tae Hoon Oh

Division of Gastroenterology, Department of Internal Medicine, Inje University College of Medicine, Sanggye Paik Hospital, Seoul, Korea

\section{Summary}

Dent et $\mathrm{al}^{1}$ reported a study entitled "Accuracy of the diagnosis of GORD by questionnaire, physicians and a trial of proton pump inhibitor treatment: The Diamond Study" in the June 2010 issue of Gut.

Patients with symptoms considered by their family practitioner to be of upper gastrointestinal origin were enrolled from 6 countries ( $\mathrm{n}=308)$. The Reflux Disease Questionnaire (RDQ) was completed and a symptom-based diagnosis was made by the family practitioner. ${ }^{2}$ Placebo esomeprazole was started after the diagnosis. Gastroenterologists made a symptom-based diagnosis and then performed endoscopy and wireless 48 hour esophageal $\mathrm{pH}$ recordings with monitoring of associated symptoms; the findings were used to determine the reference standard for the presence or absence of gastroesophageal reflux disease (GERD). Symptoms were recorded during treatment with $40 \mathrm{mg}$ of esomeprazole for 2 weeks. The main outcome measure was RDQ scoring for the presence of GERD compared to the symptom-based diagnosis by family physicians and gastroenterologists.
GERD was present in 203/308 (66\%) of the patients. Only $49 \%$ of the patients with GERD reported either heartburn or regurgitation as their most troublesome symptom. The sensitivity and specificity of the symptom-based diagnosis of GERD, were $62 \%$ and $67 \%$ for the RDQ, $63 \%$ and $63 \%$ for the family practitioners, $67 \%$ and $70 \%$ for the gastroenterologists and $54 \%$ and $65 \%$ for the 2 -week trial of proton pump inhibitor (PPI) treatment.

The symptom-based diagnosis of GERD by the RDQ, family practitioners and gastroenterologists had moderate and similar accuracy. Symptom response to a 2-week course of $40 \mathrm{mg}$ of esopmeprazole did not increase the diagnostic accuracy.

\section{Comment}

Although heartburn and acid regurgitation are considered to be distinctive symptoms of GERD and current guidelines recommend making a clinical diagnosis of GERD based on these symptoms, ${ }^{3,4}$ research on the spectrum of symptoms associated with GERD and the accuracy of a symptom-based diagnosis has been limited. This study provides rigorous validation of symp-

Received: December 18, 2010 Revised: December 26, 2010 Accepted: December 26, 2010

(c) This is an Open Access article distributed under the terms of the Creative Commons Attribution Non-Commercial License (http://creativecommons. org/licenses/by-nc/3.0) which permits unrestricted non-commercial use, distribution, and reproduction in any medium, provided the original work is properly cited.

*Correspondence: Tae Hoon Oh, MD, PhD

Division of Gastroenterology, Department of Internal Medicine, Inje University College of Medicine, Sanggye Paik Hospital, 761-1 Sanggye 7-dong, Nowon-gu, Seoul 139-207, Korea

Financial support: None.

Tel: +82-2-950-8862, Fax: +82-2-950-1955, E-mail: osbbang@paik.ac.kr

Conflicts of interest: None. 
tom-based diagnostic assessment by a self-report questionnaire and physician's skills acquired by experience in clinical practice. The authors also tried to evaluate whether a 2-week trial of PPI treatment would enhance the accuracy of the symptom-based diagnosis of GERD.

This study provides novel insights into the accuracy of various diagnostic modalities for GERD. The authors reported that the sensitivity and specificity, for the diagnosis of GERD was $49 \%$ and $74 \%$, respectively, for the symptom-based diagnosis with heartburn or regurgitation as the most troublesome presenting symptom, and $71 \%$ and $44 \%$, respectively, for a 2 -week trial of standard-dose esomeprazole treatment in the subgroup of patients that reported typical GERD symptoms. Although the PPI test is accepted as a useful and sensitive tool that allows physicians to make a rapid and fairly accurate diagnosis of GERD, ${ }^{5}$ there has been conflicting results regarding its diagnostic accuracy in patients with classic GERD-related symptoms. ${ }^{6}$ Current guidelines recommend an empirical trial of a PPI to diagnose GERD in patients with typical GERD symptoms. ${ }^{3,4}$ However, there has been no consensus on the guidelines for the accurate dose and duration of PPI treatment or the optimal cutoff value for the symptom score defining a positive response. In this study, a 2-week trial of PPI treatment showed just moderate accuracy for diagnosing GERD; therefore, the authors recommended reducing apparently unwarranted confidence in the value of PPI test. In addition, endoscopy and esophageal $\mathrm{pH}$ monitoring with symptom-association probability showed limited utility for the diagnosis of GERD due to the moderate sensitivity (57\% and 66\%, respectively). The authors emphasized that although a symptom-based diagnosis by questionnaire and physicians provided less than ideal diagnostic accuracy, it was an inexpensive, immediately available option, especially in the primary care setting, when compared to the limited efficacy of other modalities for the diagnosis of GERD.

In addition, the authors reported that only $49 \%$ of patients reported heartburn or regurgitation as their most troublesome symptom and $21.2 \%$ of patients reported dyspepsia, defined as pain or discomfort in the center of the upper abdomen, as the second most troublesome symptom. In patients without GERD, heartburn and dyspepsia were observed almost as frequently. Based on these findings, an overlap in symptoms between patients with GERD and patients with dyspepsia without GERD was evident; therefore, a substantial proportion of patients presenting with dyspepsia might have GERD, even though the symptom-based diagnosis might not identify heartburn or reflux symp- toms. For example, almost $20 \%$ of patients with functional dyspepsia and a negative heartburn questionnaire had pathological esophageal acid exposure, ${ }^{7}$ and endoscopic reflux esophagitis was found in $18.8 \%$ of dyspeptic patients without heartburn or regurgitation. ${ }^{8}$ Thus, there may be a wide spectrum of presenting symptoms in patients with or without GERD in primary care, and this can be challenging for the symptom-based diagnosis. Taken together, to improve the accuracy of the diagnosis, additional studies are needed with different symptom assessment designs using questionnaire methods.

There are several points that should be considered. First, although this study has important strengths associated with the 3 independently performed symptom-based diagnostic assessments, $39.3 \%$ of enrolled patients dropped out during the study; in addition, this study was performed only in developed countries, and may not be generalized to the populations with a high prevalence of Helicobacter pylori infection.

In conclusion, this study suggests that there is no single perfect test for GERD. Currently, the best approach to the diagnosis of GERD relies on multiple steps, with the symptom-based approach.

\section{References}

1. Dent J, Vakil N, Jones R, et al. Accuracy of the diagnosis of GORD by questionnaire, physicians and a trial of proton pump inhibitor treatment: the Diamond Study. Gut 2010;59:714-721.

2. Shaw MJ, Talley NJ, Beebe TJ, et al. Initial validation of a diagnostic questionnaire for gastroesophageal reflux disease. Am J Gastroenterol 2001;96:52-57.

3. DeVault KR, Castell DO; American College of Gastroenterology. Updated guidelines for the diagnosis and treatment of gastroesophageal reflux disease. Am J Gastroenterol 2005;100:190-200.

4. Fock KM, Talley NJ, Fass R, et al. Asia-Pacific consensus on the management of gastroesophageal reflux disease: update. J Gastroenterol Hepatol 2008;23:8-22.

5. Gasiorowska A, Fass R. The proton pump inhibitor (PPI) test in GERD: does it still have a role? J Clin Gastroenterol 2008;42:867874.

6. Numans ME, Lau J, de Wit NJ, Bonis PA. Short-term treatment with proton-pump inhibitors as a test for gastroesophageal reflux disease: a meta-analysis of diagnostic test characteristics. Ann Intern Med 2004;140:518-527.

7. Tack J, Caenepeel P, Arts J, Lee KJ, Sifrim D, Janssens J. Prevalence of acid reflux in functional dyspepsia and its association with symptom profile. Gut 2005;54:1370-1376.

8. Song HJ, Choi KD, Jung HY, et al. Endoscopic reflux esophagitis in patients with upper abdominal pain-predominant dyspepsia. J Gastroenterol Hepatol 2007;22:2217-2221. 